\title{
Lactancia materna exclusiva y participación en la vida diaria: una perspectiva ocupacional de la maternidad
}

\author{
Amamentação exclusiva e participação na vida cotidiana: \\ uma perspectiva ocupacional da maternidade \\ Exclusive breastfeeding and participation in daily life: an occupational \\ perspective of motherhood
}

\author{
Valeria Meline-Quiñones ${ }^{\mathrm{a}}$ (D), Pía Rodríguez-Garrido ${ }^{\mathrm{b}}$ (D), Inmaculada Zango-Martin ${ }^{\mathrm{c}}$ (D) \\ ${ }^{a}$ Universidad San Sebastián, Concepción, Chile. \\ bUniversidad de Barcelona, Barcelona, España. \\ 'Escola Universitària d’Infermeria i Teràpia Ocupacional de Terrassa, Terrassa, España.
}

Cómo citar: Meline-Quiñones, V., Rodríguez-Garrido, P., \& Zango-Martin, I. (2020). Lactancia materna exclusiva y participación en la vida diaria: una perspectiva ocupacional de la maternidad. Cadernos Brasileiros de Terapia Ocupacional. 28(1), 86-110. https://doi.org/10.4322/2526-

8910.ctoAO1871

\begin{abstract}
$\underline{\text { Resumen }}$
Introducción: La lactancia materna exclusiva está inmersa en un intenso debate. Por un lado, se considera que este fenómeno gira en torno a discursos vinculados a modelos biomédicos, sociales y patriarcales impactando en el rol de la mujer y, por otro lado, existe un movimiento social que aboga por este mandado de la maternidad. Si bien la literatura indica que el hecho de amamantar influye en la participación ocupacional- tanto en la diversidad como en la significación de las actividades cotidianas- no existe suficiente evidencia al respecto. Objetivo: Comprender el impacto de la lactancia materna exclusiva en la participación ocupacional de un grupo de mujeres chilenas. Método: Estudio exploratorio de carácter cualitativo realizado mediante un muestreo intencional con diez mujeres madres chilenas. Se utilizó la entrevista semiestructurada para la recogida de información. Los datos fueron analizados a través del proceso de codificación del contenido. Resultados: Las narrativas de las mujeres madres en torno al impacto de la lactancia materna exclusiva en la implicación ocupacional, aluden al amamantamiento como: ocupación significativa y/o impositiva generando alteraciones en la transición ocupacional de la maternidad y la elección de nuevas ocupaciones. La influencia del contexto sociosanitario que explica y consolida este fenómeno. Finalmente, los roles de mujer madre o madre mujer que invitan a re-construir y re-pensar las nuevas identidades ocupacionales y roles de
\end{abstract}


género asociados al amamantamiento. Conclusión: Se puede establecer una relación directa entre la lactancia materna exclusiva y la participación ocupacional de las mujeres, por ello es fundamental comprender la construcción cultural de este fenómeno y sus implicaciones personales, ocupacionales y contextuales.

Palabras clave: Lactancia Materna, Terapia Ocupacional, Identidad de Género, Actividades Cotidianas.

\section{$\underline{\text { Resumo }}$}

Introduçáo: $\mathrm{O}$ aleitamento materno exclusivo está imerso em um amplo debate. Por um lado, este fenômeno é considerado a partir de discursos relacionados aos modelos biomédicos, sociais e patriarcais impactando os papéis ocupacionais das mulheres; por outro lado, existe um movimento social que o defende como um mandato de gênero da maternidade. Já a literatura indica que a amamentação de fato influencia a participação ocupacional - tanto na diversidade quanto na importância das atividades cotidianas, demonstrando provas suficientes a este respeito. Objetivo: Compreender o impacto do aleitamento materno exclusivo na participaçáo ocupacional de um grupo de mulheres chilenas. Método: Trata-se de um estudo exploratório de natureza qualitativa, com amostragem intencional de dez mães chilenas. Utilizou-se entrevista semiestruturada para a coleta de informaçóes. Os dados foram analisados através do processo de codificação. Resultados: As narrativas das mulheres mães, em torno do impacto do aleitamento materno exclusivo na implicação ocupacional referem à amamentação como: ocupação significativa elou impositiva, gerando alteraçóes na transição ocupacional da maternidade e da escolha de novas ocupaçóes. A influência do contexto sócio sanitário que explica e estabelece esse fenômeno. Finalmente, os papéis como mulher mãe ou mäe mulher nos convidam a reconstruir e repensar novas identidades ocupacionais e os papéis de gênero associados com a amamentação. Conclusão: Foi possível estabelecer uma relação direta entre o aleitamento materno exclusivo e a participaçáo ocupacional das mulheres, por isso é fundamental compreender a construção cultural deste fenômeno e suas implicaçôes pessoais, ocupacionais e contextuais.

Palavras-chave: Aleitamento Materno, Terapia Ocupacional, Papel de Mulher, Atividades Cotidianas.

\section{$\underline{\text { Abstract }}$}

Introduction: Exclusive breastfeeding is immersed in a debate. On the one hand, is considered that this phenomenon revolves around speeches related to models biomedical, social and patriarchal impacting on the role of women and on the other hand, there is a social movement which advocates this errand of motherhood. While the literature indicates that the fact breastfeeding influences the occupational participation-both in the diversity and in the significance of daily activities- there is enough evidence in this respect. Objective: To understand the impact of exclusive breastfeeding in a group of Chilean women occupational participation. Method: exploratory study of qualitative nature carried out by intentional sampling with ten Chilean mothers. The semistructured information collection interview was used. Data were analysed through the encoding process. Results: The narratives of the mothers around the impact 
of exclusive breastfeeding in occupational engagement, refer to as breastfeeding: occupation imposition or significant generating changes in the occupational transition of the maternity and the choice of new occupations. The influence of the context public health that explains and consolidates this phenomenon. Finally, roles as mother women or women mother inviting you to rebuild and re - think new occupational identities and gender roles associated with breastfeeding. Conclusion: To establish a direct relationship between exclusive breastfeeding and the occupational participation of women, therefore it is essential to understand the cultural construction of this phenomenon and its implications, occupational, personal and contextual.

Keywords: Breastfeeding, Occupational Therapy, Gender Identity, Activities of Daily Living .

\section{Introducción}

No es posible abordar el tema de la Lactancia Materna sin entrar en un debate tensionado y polarizado desde las distintas esferas. Así, las posibilidades de encuentro se reducen considerando las experiencias y las representaciones sociales de las mujeres que, por un motivo u otro, abogaron, rechazaron o se vieron obligadas a llevar a cabo la lactancia materna exclusiva (LME) como mecanismo único de alimentación para sus hijos e hijas.

En ese sentido, Beatriz Gimeno (2018) describe que la lactancia materna debe ser abordada desde un entramado de significaciones y preceptos que permitan, por un lado, reflexionar sobre el sentido común del pecho, y, por otro lado, sobre el amamantamiento. Así mismo y a partir del mandato hegemónico que las mujeres que lactan están sujetas en la actual sociedad, Ibone Olza (2013) enfatiza en que la leche materna es el mejor alimento para los bebés, pero dar el pecho no es siempre lo mejor.

Cuando nos referimos a la alimentación mediante LME, apelamos al acto de dar al lactante leche materna de forma única sin incorporar a la alimentación agua u otros alimentos, con excepción de medicamentos, soluciones de rehidratación oral y vitaminas (Barrientos-Delgado, 2005; Chale et al., 2016).

En Chile, al finalizar la década de los ochenta, sólo el 5\% de las madres amamantaban a sus hijos/as de forma exclusiva hasta los seis meses de edad. Esta cifra alertó a las autoridades sanitarias chilenas de esa época lo que provocó un impulso en el desarrollo de una estrategia junto a otras organizaciones públicas y privadas, para incentivar y fortalecer la práctica de la lactancia materna (Centro de Investigación Periodística Chile, 2016). Después de intensas movilizaciones sociales a favor de la lactancia materna, en el año 2011 se aprueba la ley 20.545, la cual establece el derecho a la prolongación del permiso postnatal seis meses posteriores al nacimiento del recién nacido (Centro de Investigación Periodística Chile, 2016). Esta ley marca un precedente en materia de derechos sexuales y reproductivos de la mujer chilena, ya que, en el año 1925, éste era de sólo veinte días (Superintendencia de Seguridad Social, 2016). De este modo, la regulación antes mencionada se cińe a lo estipulado por la Organización Mundial de la Salud (OMS) la cual recomienda la LME desde el 
nacimiento del recién nacido hasta los seis primeros meses de vida como mínimo, siendo complementaria hasta los dos años o más (Organización Mundial de la Salud, 2017).

Desde ahí, comprendemos que la LME como acto de alimentación requiere disponibilidad absoluta de la mujer. Según la literatura, los primeros seis meses de vida del lactante las tomas de leche se realizan aproximadamente cada 2,2 horas el primer trimestre y hasta cada 3,3 horas a los 6 meses de vida (Ovies-Carvallo et al., 1999; Fallon et al., 2016; Moore et al., 2016).

La interdependencia reflejada en el acto de lactar entre la mujer y el recién nacido, en tanto comprende un requerimiento mutuo de vincularse desde una lógica biopsicosocial albergando características biológicas, nutritivas, psicológicas, emocionales y socioculturales (Gutman, 2014; Massó-Guijarro, 2013), ha quedado invisibilizada desde la óptica sanitaria e investida de las características nutritivas que involucra la LME. Este planteamiento, no aborda el componente cultural que el acto de lactar involucra en nuestra sociedad situándose la lactancia desde una comprensión capitalista y sobre todo patriarcal, inclusive siendo esta una decisión propia de la mujer (Gimeno, 2018).

Desde ahí, se reconoce que este conflictivo escenario puede generar repercusiones en las mujeres madres a nivel de salud mental, entendiéndose esta última, como el estado de bienestar en el cual el individuo es consciente de sus propias capacidades, puede afrontar las tensiones normales de la vida, puede trabajar de forma productiva y fructífera y es capaz de hacer una contribución a su comunidad (Organización Mundial de la Salud, 2019).

Esta compleja situación, da respuesta a las condiciones sociales y culturales en las cuales se desarrolla el acto de maternar y criar en la actual sociedad. Así, el ejercicio de la maternidad en esas condiciones precariza y supedita a las mujeres, en tanto existe un rol de género femenino que guía y supervisa su actuar y comportamiento (Llopis, 2015). Esto conlleva, de acuerdo con lo planteado por Llopis (2015), un impacto directo en el ámbito laboral de las mujeres no sólo produciéndose situaciones de conflicto de intereses entre el rol de madre y el rol laboral, sino también con respecto a una precarización de los puestos de trabajo con lo que esto conlleva.

De este modo, surge la categorización de la "mala madre", mujeres que, ante los ojos de la sociedad, son interpeladas por no cumplir el mandato hegemónico del género femenino, el cual implica entre otras características, el sacrificio innato por sus hijos e hijas, y la participación ocupacional ideal de la lactancia materna ante cualquier vicisitud que esta conlleve (Molina, 2006; Llopis, 2015; Pérez \& Moreno, 2017; Gimeno, 2018).

Por consiguiente, considerar el entramado de significaciones y mandatos de la lactancia materna es clave para comprender las dificultades en la implicación ocupacional- tanto en la diversidad ocupacional como en la participación en contextos vitales significativos de las mujeres- y, por tanto, el impacto en su estado de salud y bienestar, así como argumenta Montero et al. (2004), al describir la importancia del impacto que produce amamantar en la salud mental de las mujeres.

La lactancia materna tiene importantes efectos en el bienestar individual, familiar y social, de modo que hay extensa literatura sobre los diversos beneficios que la LME conlleva. En ese sentido, los estudios ratifican sus beneficios en términos nutritivos y 
psicológicos, respondiendo al efecto tranquilizador y de bienestar que produce en la mujer gracias a la liberación de oxitocina y de endorfinas durante la succión del pezón por parte del lactante, teniendo como consecuencia una menor probabilidad de sufrir depresión postparto (Aguilar-Palafox \& Fernández-Ortega, 2007; Moore et al., 2016).

No obstante, las dificultades en materia de salud mental para las mujeres, devienen e incrementan principalmente, cuando los factores socioculturales, afectan y perjudican el proceso fisiológico de la lactancia materna, ejemplo de ello se aprecia en casos de gestaciones no planificadas o no deseadas, bajo o nulo apoyo familiar, ausencia de la figura del "padre" del recién nacido, violencias de género presentes en su entorno, así también, en condiciones adversas caracterizadas por cambios psicológicos de la mujer previo o durante el proceso de gestación y puerperio (Pino et al., 2013; Hidalgo \& Menéndez, 2003).

Atendiendo a la complejidad del proceso de la LME desde lo cotidiano y situado, evidenciamos su repercusión en la participación ocupacional de las mujeres.

De acuerdo con Kennedy \& Davis (2017), la participación ocupacional es un fenómeno complejo, subjetivo y multifacético que alude al hecho de comprometerse o implicarse- tanto por elección como por necesidad- en ocupaciones o actividades de la vida diaria- como son el autocuidado, la productividad y el ocio- y de qué modo esto impacta en la salud el bienestar de las personas. Pero, es fundamental tener en cuenta que la participación ocupacional, aspecto central de la disciplina de terapia ocupacional, implica considerar diferentes aspectos como son: las demandas de la actividad, las destrezas y lo patrones de ejecución, las características de la persona, el contexto y el entorno, así como las áreas de ocupación (American Association of Occupational Therapy, 2014). Por lo tanto, considerar el contexto sociocultural de las mujeres que optan por la LME es esencial para comprender las implicaciones que este fenómeno tiene en su participación ocupacional y por lo tanto en su salud y bienestar.

De este modo, todos los cambios biológicos, psicológicos y sociales relacionados con mantener o terminar con la LME son imprescindibles para entender sus repercusiones en la participación ocupacional. Asimismo, existe una estrecha relación entre el significado personal, la valoración social y familiar de la actividad (Doble \& Santha, 2008). Estos significados se manifiestan en los sentimientos, las emociones, los pensamientos y las acciones relacionadas con la ocupación significativa (Kielhofner, 2011).

La maternidad, como ocupación, implica aspectos físicos, emocionales y socioculturales que de forma directa son determinantes en la adaptación y en la participación en las actividades cotidianas (Poole et al., 2009). En ese sentido, se espera que el proceso de lactancia materna sea natural, fácil e instintivo, pero la realidad es compleja, requiere de aprendizaje y, sobre todo, de un proceso de transición ocupacional que implica el manejo de demandas y expectativas socioculturales que no pueden ser ignoradas para poder comprender la complejidad de este fenómeno (Spano-Nakano et al., 2007).

Esta tensión en la participación ocupacional de las mujeres madres desde el marco establecido, justificado desde el rol materno y las funciones relacionadas con el cuidado y necesidades del recién nacido, resulta -la mayoría de las veces- en el abandono de sus propias necesidades y deseos (Cantero Garlito et al., 2015). En ese sentido, Loukas (1992) señala que, en la práctica de terapia ocupacional, debemos ser conscientes del 
aumento de la carga de trabajo sobre las mujeres, así también de las necesidades de todos los miembros de la familia, promoviendo de esta manera responsabilidades compartidas, situación que se contrapone a la realidad vivenciada desde el mandato cultural que suponen los roles de género en nuestra sociedad (Lagarde, 1996).

En ese sentido, considerar la lactancia materna y su impacto en la participación ocupacional desde una perspectiva de género, favorece una lectura crítica y reflexiva sobre la complejidad de la maternidad desde el punto de vista ocupacional y el entramado en el cual se sitúan las mujeres madres en la sociedad. Sin embargo, y desde la óptica de las mujeres que optan por la LME, las condicionantes culturales no acaban de dar respuestas a las dinámicas sociales entre las cuales interactúan, desde ahí, la relevancia de leer en clave de género (Lagarde, 1996) estos comportamientos en torno a la maternidad y su impacto en lo cotidiano, ya que nos permite develar y problematizar las conflictivas relaciones en las cuales se ven inmersas las mujeres madres que optan por esta ocupación (Olza, 2013; Gimeno, 2018).

A partir del contexto presentado, el objetivo de este estudio plantea comprender desde una perspectiva ocupacional la lactancia materna exclusiva identificando su impacto en la participación de ocupaciones significativas para un grupo de mujeres chilenas.

\section{Método}

\subsection{Diseño metodológico}

El marco metodológico de la presente investigación se desarrolló bajo las comprensiones del paradigma cualitativo, ya que nos permitió reconocer la existencia de múltiples realidades subjetivas, comprendiendo y analizando la complejidad de la experiencia humana y sus significados (Vázquez Navarrete et al., 2006). Teniendo en cuenta que el impacto de la lactancia materna en la participación ocupacional de las mujeres madres es un tema complejo, es determinante considerar las diferentes perspectivas para su comprensión, ya que este fenómeno no puede ser abordado considerando nexos causales directos.

En ese sentido, la metodología cualitativa implica una visión panorámica del cuerpo de estudio, variable y nunca objetiva, con la finalidad de comprender las dinámicas sociales de los/as sujetos/as en sus propios contextos, propiciando una dialéctica flexible entre el/la investigador/a y el/la sujeto/a de estudio que permita aumentar la comprensión de la realidad en la cual se trabaja (Dávila, 1995; Vázquez Navarrete et al., 2006).

\subsection{Diseño muestral}

Las características del diseño muestral se plantearon de acuerdo a los objetivos de esta investigación. Todas las participantes fueron seleccionadas a través de un muestreo intencional que permitió descubrir y describir el fenómeno estudiado con mayor profundidad. Así, se considera importante destacar la representatividad discursiva de las sujetas entrevistadas y sus significados. 
La muestra del estudio estuvo conformada por diez mujeres chilenas que utilizaron la lactancia materno exclusivo como único método de alimentación para sus hijos e hijas. Así también, se encontraban gozando del periodo de licencia maternal postnatal, la cual tiene una duración extensible -en el caso de Chile- hasta seis meses post parto (Biblioteca del Congreso Nacional de Chile, 2016). El estudio se realizó en Chile, país ubicado en Sudamérica, dividido geográficamente en dieciséis regiones. Las diez mujeres que accedieron a participar y que cumplían con los criterios de inclusión, habitaban en distintas regiones del país, es así como una de ellas vivía en la primera región de Tarapacá (norte de Chile), tres de ellas vivían en la región Metropolitana (centro de Chile), cinco de ellas vivían en la octava región del Bio-Bio (sur de Chile), y una de ellas vivía en la décima región de Los Lagos (extremo sur de Chile).

Los criterios de inclusión de las participantes fueron: ser mujer, madre, residente en Chile, mayor de edad, que utilizaran la lactancia materna exclusiva como único alimento para sus hijos e hijas, y que una vez ocurrido el parto ya sea vaginal o cesárea, tanto la madre como el recién nacido se fueran en conjunto a su domicilio.

Por otra parte, los criterios de exclusión fueron: mujeres que se encontrasen alimentando a sus hijos o hijas con métodos mixtos, es decir, lactancia materna junto a sustitutos alimenticios, que hayan permanecido hospitalizadas al momento del postparto y de la entrevista, enfatizamos en esta condición, ya que al quedar hospitalizada la madre y el recién nacido, este último no permanece las 24 horas del día en alojamiento conjunto, generando que en muchas ocasiones, se le administre alimentación por fórmula en vez de lactancia materna. Y, finalmente, que no hayan decidido participar del estudio, por lo tanto, no firmaran el consentimiento informado.

La aproximación a las participantes se realizó mediante un llamado público a través de redes sociales (Facebook). En respuesta a ello, se procedió a contactar vía telefónica a las mujeres que cumplían con los criterios de inclusión del estudio.

\subsection{Técnica de recogida de datos}

La técnica de recogida de información utilizada en este estudio fue la entrevista semiestructurada, ello con la finalidad de obtener información que permitiera comprender las perspectivas y los significados que tienen las participantes acerca del fenómeno en cuestión.

Se elaboró un guión de preguntas direccionado a responder el objetivo de la investigación, no obstante, surgieron preguntas fuera del itinerario establecido, las cuales se vinculan al rol de la figura "paterna" y los aspectos sociales que este involucra.

Para concertar las entrevistas, se realizó una llamada telefónica a las participantes para coordinar la logística del encuentro. Una vez concretada la cita, se procedió a explicar el consentimiento informado y a su posterior firma y grabación de este (en el caso de la entrevista por video-llamada). Finalmente, se realizaron cinco entrevistas presenciales y cinco entrevistas a través de video-llamada. Esto último, debido a la distancia geográfica que las participantes se encontraban en relación con la entrevistadora. 
Las entrevistas realizadas de manera presenciales como a través de video-llamada fueron grabadas en formato de audio teniendo una duración de una hora y quince minutos en promedio.

Para identificar a las participantes se les asignó el código P de participante y un número -iniciando con 1 - correspondiente al orden en el que las entrevistas fueron realizadas (Tabla 1$)$.

Tabla 1. Características de las participantes del estudio.

\begin{tabular}{ccccccccc}
\hline $\begin{array}{c}\text { Participante } \\
\text { (P) }\end{array}$ & Edad & Pareja & $\begin{array}{c}\mathbf{N}^{\circ} \text { de } \\
\text { Hijos/as }\end{array}$ & $\begin{array}{c}\text { Tipo de } \\
\text { familia }\end{array}$ & $\begin{array}{c}\text { Edad del } \\
\text { lactante }\end{array}$ & $\begin{array}{c}\mathbf{N}^{\circ} \text { tomas } \\
\text { diarias }\end{array}$ & $\begin{array}{c}\text { Experiencia } \\
\text { previa de } \\
\text { LME }\end{array}$ & $\begin{array}{c}\text { Región de } \\
\text { Chile de la } \\
\text { participante }\end{array}$ \\
\hline P 1 & 29 & $\mathrm{Si}$ & 2 & Extensa & 5 meses & 8 & $\mathrm{Si}$ & Biobío \\
\hline P 2 & 27 & $\mathrm{Si}$ & 2 & Extensa & 7 días & 15 & $\mathrm{Si}$ & Biobío \\
\hline P 3 & 30 & $\mathrm{Si}$ & 2 & Nuclear & 3 meses & 6 & $\mathrm{Si}$ & Metropolitana \\
\hline P 4 & 23 & $\mathrm{Si}$ & 1 & Nuclear & 4 meses & 8 & No & Biobío \\
\hline P 5 & 31 & $\mathrm{Si}$ & 2 & Nuclear & 7 meses & 20 & $\mathrm{Si}$ & Biobío \\
\hline P 6 & 30 & $\mathrm{Si}$ & 1 & Nuclear & 5 meses & 7 & No & Los lagos \\
\hline P 7 & 31 & $\mathrm{Si}$ & 1 & Nuclear & 5 meses & 13 & No & Tarapacá \\
\hline P 8 & 31 & $\mathrm{Si}$ & 1 & Nuclear & 2 meses & 8 & No & Metropolitana \\
\hline P 9 & 23 & $\mathrm{No}$ & 3 & Extensa & 4 meses & 10 & $\mathrm{Si}$ & Biobío \\
\hline P 10 & 37 & $\mathrm{Si}$ & 2 & Nuclear & 2 meses & 8 & $\mathrm{Si}$ & Metropolitana \\
\hline Promedio & $\mathbf{2 9 , 2}$ & & $\mathbf{1 , 7}$ & & $\mathbf{4}$ meses & $\mathbf{1 0 , 3}$ & & \\
\hline
\end{tabular}

Las mujeres entrevistadas fluctuaban entre los 23 y 37 años, siendo la media de edad de 29 ańos. Los lactantes al momento de la investigación tenían entre siete días y siete meses. Todas las madres que tenían más de un hijo poseían experiencia previa en lactancia materna exclusiva.

Siete mujeres residen con su familia nuclear conformada por sus parejas e hijos. La otras tres entrevistadas residen con su familia nuclear extensa. Nueve de las diez entrevistadas se encuentra conviviendo con su pareja, a excepción de una de ellas que compone una familia monoparental junto a sus padres.

\subsection{Análisis de los datos}

El proceso de análisis de los datos dentro de la investigación cualitativa es un proceso en continuo progreso, dinámico y creativo que se dá de forma simultánea con la recolección, la codificación, la interpretación y la escritura narrativa de los datos (Taylor \& Bodgan, 1987; Coffey \& Atkinson, 2003).

Teniendo esto en cuenta, el proceso de codificación es un proceso analítico particular a través del cual se segmentan y reorganizan los datos en códigos o categorías, los cuales son el soporte para interpretar los datos derivados de la investigación. El proceso de codificación, de acuerdo con Coffey \& Atkinson (2003) se caracteriza por ser dinámico y tener como finalidad establecer conexiones con diferentes segmentos de los datos con conceptos y categorías en función de alguna propiedad o elemento común. 
A partir de lo descrito anteriormente, y junto a la revisión de la literatura en apoyo a las narrativas de las participantes entrevistadas, se elaboraron tres categorías principales de análisis (ver Figura 1): lactancia materna exclusiva como ocupación significativa y/o impositiva, lactancia materna exclusiva en el contexto socio-sanitario y mujer madre o madre mujer.

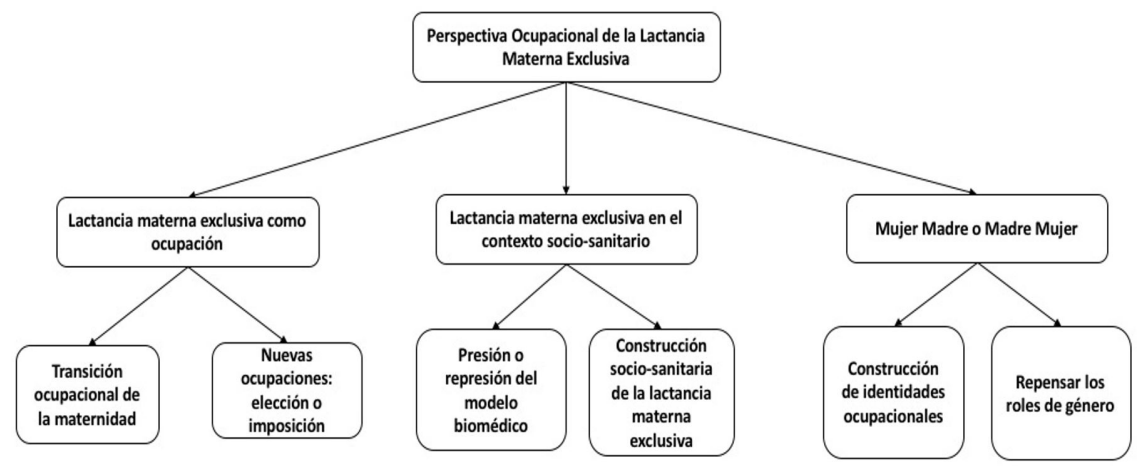

Figura 1. Mapa conceptual sobre la Perspectiva Ocupacional de la Lactancia Materna Exclusiva.

Estas categorías aluden a los conceptos que emergieron en el proceso de codificación desde un análisis del contenido orientado semánticamente. De este modo, emergieron a la vez, seis subcategorías de análisis, estas fueron: transición ocupacional de la maternidad, nuevas ocupaciones: elección o imposición, presión o represión del modelo biomédico, construcción socio-sanitaria de la lactancia materna exclusiva, construcción de identidades ocupacionales y repensar los roles de género.

\subsection{Consideraciones éticas}

Previo a la realización del trabajo de campo, se contó con la aprobación del comité de ética de la Universidad de Castilla-La Mancha para aplicar el consentimiento informado tipo utilizado en investigaciones con seres humanos de acuerdo a los criterios del Máster de Terapia Ocupacional en Salud Mental en el que se enmarca dicha investigación.

Al inicio de cada entrevista, se hizo lectura del consentimiento informado por parte de la investigadora, quien explicaba brevemente los objetivos de la investigación y el uso que se dará a las entrevistas, a su vez, explicó a las participantes que sus datos personales serán resguardados en el anonimato y que podrán abandonar la investigación cuando lo consideren pertinente, dicho consentimiento fue firmado por las mujeres madres con quienes se realizó la entrevista presencial y fue grabado en audio en el caso de las entrevistas que fueron realizadas mediante video-llamada.

Debido al resguardo de los datos personales de las entrevistadas, su participación será mencionada como: participante y los nombres de sus hijos e hijas serán mencionados como bebé o lactante, así también, el nombre de sus parejas será resguardado. 


\subsection{Criterios de calidad del estudio y rigor metodológico}

El criterio de calidad del estudio se sustentó en el criterio de credibilidad, el cual permitió manifestar la congruencia entre los resultados obtenidos y la perspectiva teórica que sustenta el fenómeno a analizar. En relación con el rigor metodológico, se procedió a la triangulación de las investigadoras del presente estudio, caracterizado por su diversidad profesional, y, por tanto, su posición frente al fenómeno estudiado. Así, las investigadoras- dos terapeutas ocupacionales y una matrona- ofrecieron diferentes perspectivas tanto desde comprensiones sanitarias, sociales y culturales de la ocupación, como desde una perspectiva perinatal, salubrista y de género. Esto es considerado un criterio que enriquece y propicia calidad al estudio, ya que genera una visión transdisciplinar del fenómeno estudiado. Del mismo modo y para fortalecer el criterio de validez metodológica, se utilizó la Guía de Revisión para Estudios Cualitativos: Standard for Reporting Qualitative Research (SRQR).

\section{Resultados}

Para proceder al análisis de los resultados -relacionados con el impacto de la LME en la participación en las ocupaciones de las mujeres madres- se establecieron categorías y subcategorías en relación con la conceptualización y la comprensión de las entrevistadas sobre la perspectiva ocupacional de la LME. Así, la perspectiva ocupacional de la LME permite identificar tanto el impacto en las ocupaciones diarias de las participantes, como la participación en ocupaciones significativas.

De este modo y de acuerdo con las narrativas analizadas, presentaremos a continuación las categorías y subcategorías emergentes del estudio.

\subsection{Lactancia materna exclusiva como ocupación}

La LME no es la única ocupación desempeñada por las participantes del presente estudio ya que también realizan otras ocupaciones relacionadas con la situación de maternidad. Sin embargo, la LME se ha planteado como ocupación considerando de qué modo las participantes, bien respondiendo a criterios de elección, imposición o necesidad, y marcadas de forma directa por el contexto sociocultural en el que están inmersas, se implican dicha actividad significativa. Así, de acuerdo con las narrativas de las participantes entrevistadas, optar por la lactancia materna exclusiva como único medio de alimentación para sus hijos e hijas, es vista como una ocupación significativa, y en algunas ocasiones, como una ocupación impositiva, dependiendo de sus experiencias y significados. En ese sentido, emergen dos subcategorías relacionadas con esta dimensión: Transición ocupacional de la maternidad y Nuevas ocupaciones: elección o imposición.

\subsubsection{Transición ocupacional de la maternidad}

En el proceso de transición ocupacional, las participantes identifican como ocupaciones más significativas aquellas relacionadas con el autocuidado, situaciones cotidianas que han tenido que modificar para ejercer el rol de madre, condición que anterior a sus gestaciones no habían experimentado. 
La ducha, porque eso son los 2- 3 minutos que tenía para mi, para mi son 2-3 minutos que son para mí, para nadie más, entonces realmente hay dias que no lo tengo y para mi eso es horrible, es horrible no poder ducharme y no por el sentido de que uno se sienta hedionda, sino que son esos 2 minutos que son mios, mios, mios, para mi sola en el baño jaja porque lo demás no estoy nunca sola (P5).

De acuerdo con las narrativas de las mujeres, las dificultades para participar e involucrarse en ocupaciones significativas están vinculadas a sentimientos negativos y por lo tanto, tienen un impacto tanto en su autoconcepto como en el estado de su salud mental.

Optar por la lactancia materna exclusiva como fuente de alimentación para sus hijos e hijas está vinculado en muchas ocasiones, a situaciones de angustia, nervios y preocupación. Sin embargo, algunas entrevistadas, señalan que estas emociones vinculadas a la lactancia materna son temporales y que no implican necesariamente, el sentimiento de abandono de esta.

Ya mira, al principio era angustia mucha angustia, porque no sabia si estaba tomando bien el pecho, si quedaba bien, si mi leche era buena... Después estrés mucho estrés y no sabia cómo organizarme, no sabía si estaba bien sentada para darle pecho, no me podía acomodar, eso todavia me cuesta, hay veces que la leche me disminuye mucho, hace dos días tenía muy poca leche, al momento de extraerme no me salia mucha, entonces te preocupas, te pones nerviosa y eso yo creo que te juega en contra. Todavía no llega el momento en que sienta que es placentero... (P3).

En ese sentido, se puede observar que estas emociones -principalmente el miedo y la angustia- se agudizan al inicio del proceso de lactancia materna. Si bien algunas de las participantes no tienen experiencia en este ámbito y esto puede influir en la incertidumbre con respecto a si los lactantes están recibiendo suficiente alimento, otras participantes expresan estas emociones pese a tener experiencias previas en torno a la lactancia materna. Estos aspectos son fundamentales para comprender el impacto de la lactancia materna en la salud mental de las mujeres madres.

Otro de los aspectos seńalados por una de las participantes hace referencia a la situación de soledad e incertidumbre experimentados al inicio del proceso de lactancia materna, enfatizando en el tiempo y conocimiento como condiciones necesarias para adaptarse a los nuevos ritmos ocupacionales.

En un inicio me sentía demasiado sola, yo creo que a todas nos pasa, las que tenemos un bebé por primera vez, no sabia bien que le pasaba, como calmarlo, me desesperaba sola con mi pareja, los dos primera vez, entonces en un inicio fue muy dificil, además que era muy demandante de la pechuga los primeros meses, entonces era todo el día abi en la pechuga, mucho rato, muchas veces, entonces eso fue lo más complejo al principio, ahora ya no porque, ya tiene 5 meses, ya sé sus horarios, muchas veces que le pasa, aunque a veces no mucho pero eso fue al principio (P7). 


\subsubsection{Nuevas ocupaciones: elección o imposición}

Esta subcategoría señala el modo en que las participantes identifican las ocupaciones que se derivan de la lactancia materna y cómo éstas han sido incorporadas ya sea por elección y/o imposición en su cotidianidad. Sin embargo, casi todas ellas sostienen que el proceso de lactancia materna provoca una ruptura con su cotidianidad lo que conlleva un reajuste en todos los niveles: físico, psicológico y social. Teniendo un fuerte impacto sobre su estado de salud y bienestar.

A veces igual si, me siento súper encerrada, porque obviamente en mi vida, antes yo tenía harto... mucha actividad, salia harto en la misma universidad pertenecía a varios grupos y ahora en realidad no salgo mucho por el clima, por la misma guagüita, pero pienso que es una actividad que de cierta forma va a llevar beneficios para ella y también para mi y que en un futuro, en un par de años ya no va a estar o va a ser menos demandante entonces voy a volver a tener esas libertades (P4).

En ese sentido, las participantes expresaron que el hecho de optar por la lactancia materna exclusiva tuvo un impacto directo en su falta de implicación en actividades que para ellas eran significativas -y por ende vinculadas a su identidad- debido a la sobrecarga y agotamiento que la lactancia -como actividad-conlleva.

Si se me viene una palabra, es como hee... me siento agotada, cuando de hecho, creo que es la frase que más le digo a mi pareja, cuando me dice que te pasa negrita: jes eee estoy agotada! Si, cuando ando "pateando la perra" como se dice, es porque estoy agotada, porque tengo que cambiar pañales o porque tengo que darle leche (P1).

El ejercicio de la lactancia materna exclusiva se percibe como algo únicamente de carácter reproductivo, ya que no genera remuneración económica como un "trabajo productivo", por lo tanto, le resta importancia ante la sociedad.

[...] me aburre, a veces me aburre tener que dar leche, porque siento que en vez de estar ahi sentada dándole leche, podría estar haciendo otras cosas más productivas o arreglándome (P1).

Asimismo, las participantes manifiestan que la sobrecarga ocupacional vinculada directamente al estrés y a la frustración relacionada con el hecho de amamantar, influyen de manera negativa en su salud mental y en la percepción de sí, así como en sus relaciones íntimas de pareja.

Emm al comienzo me pasó que estaba súper preocupada, me acuerdo que muy muy preocupada, por el tema de la lactancia, porque como habia tenido una mala experiencia con mi primer hijo, no quería volver a pasar por lo mismo, eemm eso es, bueno, fueron noches de no dormir, noches de angustia, de llanto y emm como estrés, finalmente yo creo que el estrés se reflejaba asi, por medio del llanto o discusiones constantes [con su pareja]... (P1).

Otra de las participantes, indica la lactancia materna como la mejor elección entre las otras opciones disponibles en el proceso de -alimentación- crianza, haciendo 
referencia a la paciencia que implica llevar a cabo la lactancia materna exclusiva. Así, algunas participantes aluden a la paciencia como una actitud clave dentro del proceso de crianza siendo crucial para evitar su deserción.

No sé si servirá lo del grupo de lactancia, pero yo estoy en un grupo cerrado de Facebook, que son puras mamás, entonces siempre se comenta de todo, generalmente, el tema que más sale es este, muchas apoyan la lactancia materna $y$ como que rechazan a las que dan relleno y hay otras que quieren, quieren, quieren y dicen que claro, la paciencia es el mejor amigo de la lactancia (P7).

\subsection{Lactancia materna exclusiva en el contexto socio-sanitario}

Las participantes entrevistadas señalan que en el contexto socio-sanitario la lactancia materna exclusiva se encuentra respaldada por la opinión biomédica apoyada por un modelo cultural que valida y sustenta el acto de lactar en nuestra sociedad. Esto implica considerar la lactancia como un fenómeno complejo inmerso en un contexto biomédico cuyos intereses en ocasiones ignoran el impacto de este proceso en la participación ocupacional de las mujeres y su impacto en la salud y el bienestar.

\subsubsection{Presión y represión del modelo biomédico}

Respecto a esta subcategoría, las participantes señalan el miedo como uno de los sentimientos que prima durante el proceso de lactancia. Sin embargo, el miedo está vinculado con la exigencia de querer hacer las cosas bien, cumplir con los requerimientos del lactante y poder alimentarlo de manera correcta de acuerdo con los parámetros sanitarios. Así, estas emociones impactan en la cotidianidad de las mujeres generando una vulnerabilidad que se añade a la situación de complejidad del proceso de lactancia.

[...] el miedo de no saber si lo estás haciendo bien o lo estás haciendo mal, si lo estás alimentando o no (P6).

En ese sentido, una de las participantes señala la importancia del refuerzo positivo por parte de los profesionales de la salud, ya que favorece la seguridad y elimina los sentimientos de angustia y miedo que se manifiestan durante ese proceso. Por consiguiente, la salud mental es un aspecto clave a considerar en las mujeres madre que optan por este método de crianza -alimentación-.

[...] no sé pienso yo... deberían alentarme más, además del tema informativo, también el alentarte, porque de repente me pasa que me pilló con otra mamá que tiene una niña más grande y me dice jmuy bien! Y como que no sé, te da un refuerzo positivo y eso extrañamente a uno lo hace sentir mejor, como que te hace sentir que otra persona en cierta forma te reconozca que lo estás haciendo bien, mucho más a una mamá primeriza porque uno no sabe cómo lo está haciendo, entonces para uno es como todo nuevo, extraño, entonces que alguien te dé un refuerzo en la buena onda no más, de decirte que lo estás haciendo bien, es como 
súper positivo. Quizás eso igual lo deberian hacer más las personas de salud, si ven que tu guagüita va bien, que está creciendo, está sanito y todo eso (P7).

De acuerdo con lo anterior, se evidencia el carácter controlador e higienizador que ejerce el personal socio-sanitario ante la búsqueda de aceptación y aprobación por parte de las mujeres que lactan. Esta situación está sustentada desde las dinámicas de poder establecidas en los espacios sanitarios, expresadas en los actos de infantilización y asistencialismo hacia las mujeres y su entorno.

[...] sí, me sentía presionada, de repente venía la matrona y te ponia a la guagua como de una forma no muy... no como ahora vivo... entonces digo ¿porque ella lo hizo de una forma como bruta?, si no es como que uno le ponga la teta y que la guagua te agarre el pezón. Ahi me sentí como un poco presionada, ah y además que mi guagüita nació más flaca, nació de 2 kilos y medio, entonces era cómo: es que tienes que darle teta porque... y yo sabia que tenía que darle teta, sabia que para ella era mucho más importante que se alimentara luego, que quizás para una guagüita que tenía un peso normal, pero no era tan fácil y bueno no fue fácil pero en el momento si me sentí un poco presionada (P4).

En esta misma línea, otra participante agrega:

Te están dando de una u otra forma como una receta de lo que tienes que hacer y tú en la realidad te das cuenta de que no puedes hacerlo asi, entonces empiezas como con inseguridad, de que chuta, ¿lo estoy haciendo bien? ¿Mi guagua estará bien? ¿Porque me están diciendo cada 3 o 4 horas y me está pidiendo antes, entonces qué hago? ¿Le doy cuando ella quiera? ¿Cuándo ella tenga hambre? $¡ O$ lo que me está diciendo el pediatra? Entonces de una u otra forma igual te sientes insegura, igual tienes miedo, no sé, cómo que de repente sientes que no está empatizando él contigo, el pediatra creo yo. Y por lo menos yo le he dado cada vez que ella tiene hambre el pecho y ahora cada vez que la van a medir y pesar, esta perfecta entonces siento que está súper bien (P6).

Similar situación se manifiesta ante las complicaciones de la lactancia materna exclusiva y la posición tecnocrática y mecánica como respuesta de los profesionales de la salud ante esta situación. Esto a su vez genera limitaciones y restricciones en la participación de ocupaciones significativas de las mujeres madres y, por consiguiente, una ruptura en la vida ocupacional que impacta claramente en la salud y bienestar.

El primer mes era estresante, como te digo estaba angustiada porque no sabia si mi leche le hacia bien o no, trataba de darle leche de fórmula y no sabia si eso le iba a hacer bien, le iba a ayudar o no eem pero yo el primer mes te diré que trate de no darle, cómo? Le dije a la pediatra del centro, soy yo la que se siente mal, soy yo la que está comiendo mal y no quiero darle más pecho y como que se me vinieron todos encima $(\mathrm{P} 3)$.

No obstante, según explica otra de las participantes, cuando el equipo de salud se implica en el proceso de manera cercana, cálida y humana, se evidencia un impacto directo y positivo en la adhesión a la lactancia materna. 
[...] yo llegue a especialistas de lactancia, y estoy segura de que, si hubiera llegado o no hubiese llegado donde ellos, con mi primer hijo y mi segunda hija, hubiese abortado misión de lactancia, porque fue un periodo de mucha educación, de mucho aprendizaje (P1).

\subsubsection{Construcción socio-sanitaria de la lactancia materna exclusiva}

El discurso socio-sanitario más frecuente en torno a la lactancia materna exclusiva, se vincula a su carácter nutritivo beneficioso para la salud del recién nacido, en ese sentido, las participantes describen la importancia de la educación y de alimentar de esta manera:

[...] me dediqué como a informarme sobre la lactancia materna exclusiva, porque en mis dos hijos vi el cambio por ejemplo de la repercusión que tuvo en su salud hoy en día, o sea mi deducción fue por la lactancia materna (P9).

[...] los beneficios que tiene, por ejemplo, en el sistema inmunológico de ellos (P3).

Otra retórica utilizada, se relaciona con las características psicosociales que la lactancia materna exclusiva posee, en ese sentido, se comprende como favorecedora del "apego", generando un mayor y mejor vínculo entre el binomio mujer/madre y el lactante.

Para mi no es tan solo entregar alimento, si no que el vinculo, el apego que se forma, porque es bonito, encuentro que es muy lindo porque están tomando leche y como que te miran y es algo más que alimentación, es vinculo, es amor, es apego, es protección... (P9).

[...] es como una forma de apego que nada más te puede dar, mm como que... ni un acto, ni cosa, ni acto, ni situación te puede suplementar ese acto de amamantar con tu hijo (P2).

[...] pa mi la lactancia en general, tiene mucho que ver... con algo muy emocional, más allá de todo lo bueno que, ya el proceso lógico es muy mágico y cuántico, y creo que eso genera cosas emocionales muy fuertes, entonces si tú no estás tranquila... no tiene sentido y tampoco creo que vaya a ser exitosa (P8).

Sin embargo y a pesar del carácter beneficioso que tiene la lactancia materna exclusiva, aún persiste una fuerte presión social frente al hecho de amamantar en un contexto público, entendido esto como algo que está fuera de lugar, por lo que se les excluye directa o indirectamente a las mujeres que amamantan de los espacios públicos, situación que inclusive hasta ellas mismas han normalizado.

La única presión social que he sentido en relación con la lactancia es que sigue siendo muy mal visto, si yo quiero alimentar en la calle a mi hijo... eeemmm, me paso que en un restaurante que tuve que irme a un baño [para amamantar] $y$ 
claro po' con mi primer hijo... tú no tenías las herramientas como para poder increpar a esa persona po'... duele po', duele como que no sea comprendido (P1).

Uno siempre busca la polera cómoda, que uno no tenga que estar sacándose toda la polera para poder sacar la pechuga, entonces como que uno busca claro, una polerita lo más privado posible para poder sacarla cuando haya que sacarla (P9).

[...] pierde mucha... intimidad, en que te digo, porque tu tienes que sacarte la pechuga, darle pecho, donde estés, a mi ... ese no es mi problema que la gente me vea, si no que ee encuentro que tu tení como que sacarte la ropa en cualquier parte, no sé... toda la privacidad que uno tenía queda de lado (P3).

Esto pone de manifiesto la construcción social en relación a la lactancia materna y de qué modo esta ocupación está asociada a la articulación entre el discurso biomédico sobre la lactancia materna exclusiva por obligatoriedad y el discurso patriarcal hegemónico de lactar, pero a escondidas.

Por ejemplo cuando voy a dar de comer [referido al proceso de amamantar] de repente siento cuando me miran...con malas caras, es como: oye por qué lo está haciendo acá, no sé, es como más ligado a eso, siento cuando la gente me mira mal (P9).

\subsection{Mujer madre o madre mujer}

En esta tercera y última categoría de análisis, se alude al acto de anteponer, o más bien omitir, la categoría del ser mujer, por el del ser madre, situación que fragmenta a las sujetas, en tanto deben decidir por una u otra identidad.

\subsubsection{Construcción de identidades ocupacionales}

La necesidad de considerar de qué modo se lleva a cabo la construcción de identidades ocupacionales en el proceso de la lactancia materna queda reflejada en la siguiente narrativa.

Yo pienso que desesperación de repente de querer escapar de toda esta responsabilidad, de querer tener un tiempo para mi de repente, de cómo mujer y no mamá, emm es como eso, de repente si, si me siento desesperada. Puede ser agobio, porque viene en periodos cortos por ejemplo... si es como agobio (P9).

La participante hace alusión a la necesidad de considerarse mujer y no únicamente madre, lo cual plantea de qué modo la incorporación de roles ocupacionales vinculados a los roles de género cuestionan la identidad ocupacional e invitan a problematizar este aspecto desde otras perspectivas. Por otro lado, esta narrativa evidencia cómo la dificultad para involucrarse en ocupaciones está vinculado a sentimientos negativos.

En su mayoría, las participantes señalan los cambios corporales de la gestación y del proceso de lactancia como aspectos para tener en cuenta con respecto a la construcción 
de la identidad ocupacional y cómo la ambivalencia- mujer/madre o madre/mujer- está presente durante este proceso.

Para mi es chocante, no sépo, viene gente de visita y yo no voy a dejar de alimentar a mi hijo por no sacar la pechuga, pero si... ee... no sé po, por ejemplo, a mi me salieron estrías en el embarazo, y eso de sacarte... levantarte la polera para sacar el pecho, jtodos te ven! Más cuando estás recién paria, porque estas gordita, tenia estrias, las estrías no son blancas, son rojas, entonces llaman más la atención entonces es chocante para uno y para la otra persona también (P3).

La baja autoestima reflejada en los relatos de las participantes durante el proceso de lactancia materna está asociada a los cambios corporales surgidos durante la gestación y el puerperio, en ese sentido, perciben que han despreocupado su autocuidado en pro del cuidado de sus hijos e hijas.

[...] me siento como que me postergue, como que me estoy postergando en mis cosas (P3).

Sabe, que a veces me he sentido frustrada, como que he postergado mi... el ser mujer, el ser persona, porque soy dependiente de mi hijo completamente, no hago nada mio, no salgo sola a ninguna parte, a veces me frustro por eso, pero es cuando me viene la crisis. Se lo he dicho a mi pareja... me siento fea, no me he arreglado el pelo, ya no me pinto, muy poco, eso, entonces como que eso me hace sentir muchas veces triste, con rabia... (P7).

Esta semana me pinté las uñas, asi como no me las pintaba hace muchos años me las pinté como por eso, asi como para hacer algo para mi (P8).

He tenido que dejar muchas cosas de lado y eso es el tiempo, el tiempo como mujer también, como mamá, de repente no alcanzar a peinarme, quizás, o dejar de hacer otras cosas por dedicarme a estar con ella, porque requiere de tiempo y en la noche igual, es algo que yo o sea uno no duerme cuando da lactancia materna, entonces eso igual repercute en el día a dia (P9).

Así mismo, algunas participantes refieren la nostalgia que les produce el hecho de reconocerse como madres dentro de una nueva identidad y verse anuladas como mujeres por la sociedad.

Un dia me acuerdo que no paraba de llorar y fui a ver a mi perra, de verdad la sacamos de la pieza porque antes dormía acá con nosotros y la sacamos por el bebé y estaba durmiendo al lado en otra pieza y la fui a abrazar al lado porque la echaba de menos y ahi lloré como casi como magdalena (haciendo referencia al personaje biblico de Maria Magdalena) y ahi me despedi de ella pero de mi, como de mi antigua vida, porque ya el minuto de ser mamá, cargar con alguien durante toda tu vida po cachay, entonces como que eso es igual como bien fuerte po... (P8).

Ante este escenario, las participantes expresan la importancia de generar espacios de encuentros, diálogos y redes con otras mujeres madres. En ese sentido, la construcción de la identidad ocupacional desde la lactancia materna se concibe como promotora de 
la interacción social, dejando en claro que esta interacción surge a partir de las condicionantes socio-culturales en los cuales se materna hoy en día.

[...] mira ya sea el papá o sea cualquier figura, tu mamá o algo cachay, pero alguien que esté ahi al lado apañando, claro, si es el papá mucho mejor, pero en el caso de que no fuera asi, cualquier otra persona que cumpla el mismo rol como el papá, que esté tan comprometido sirve (P8).

Así, el apoyo de la comunidad se muestra como fundamental en la práctica de una lactancia materna exitosa, puesto que la colaboración de otros alivia la carga física y emocional que la mujer madre debe sortear.

Como ahora están ellos, yo si he tenido más tiempo para hacer cosas hacia mi persona, pero yo entiendo que esa regalía se da solo porque casi que estamos criando en comunidad, pero si yo... hasta hace un par de semanas atrás, el cuento era súper distinto, no tenía tiempo para mi en absoluto (P1).

En esta misma línea, otra participante sostiene:

[...] cuando yo estoy muy agobiada voy donde una vecina, que también tiene una guagua de la edad, le digo, ¿te la puedo dejar un ratito? Y cuando ella está muy agobiada también hace lo mismo (P5).

Esto implica una construcción de la identidad ocupacional centrada en la comunidad y no tanto en la individualidad. De acuerdo con una de las participantes, otro grupo de soporte fundamental durante el proceso de lactancia materna son los grupos de lactancia, principalmente en modalidad "on line". Estas comunidades virtuales, son utilizadas principalmente para encontrar información y acompańamiento de otras mamás en las distintas fases de la lactancia materna:

Encuentro que ha sido súper bueno porque me siento como empoderada con la información que antes no tenía de la lactancia y cualquier duda yo consulto y están todas súper siempre dispuestas a responder las dudas que uno tiene (P9).

Del mismo modo, otras participantes señalan la relevancia de participar en actividades que las vinculen a mujeres que están experimentando la misma situación como una manera de establecer una relación de soporte y acompañamiento en el proceso:

Me hace sentir bien, porque hablo... hay gente que está pasando por lo mismo que yo, entonces comparto ideas (P6).

[...] que yo misma pueda ayudar a otras personas y decirles mira asi se hace el banquito de leche, no te preocupes, déjalo aqui en esta parte del refrigerador en este frasquito, como que si, me siento súper bien con eso, en cuanto a eso de la información que yo sé, de poder explicarlo y enseñarlo a otras personas (P8). 


\subsubsection{Repensar los roles de género}

La lactancia materna exclusiva está constantemente imbricada a los roles de género, a partir de ello, muchas mujeres han visto omitida su identidad de mujer por la categoría de madre, mas, desde comprensiones que apelan a las "buenas" o "malas madres", se les obliga e impone un modelo hegemónico de cómo llevar a cabo la ocupación de lactar.

A partir de ahí, la división sexual de las ocupaciones quedan entrampadas en la dualidad de lo reproductivo/privado y productivo/público.

[...] cuando pienso en trabajar automáticamente lo que se te viene es el tema de la leche, de cómo voy a hacer para alimentarla, porque yo no le quiero dejar de dar leche entonces ee si los trabajos fueran más flexibles para poder alimentar, eso sería mucho más tranquilizador para nosotros po, me refiero como para las mamás que dan lactancia, porque la vida laboral no es amigable y no es compatible con la lactancia, porque en qué trabajo te van a dejar. emm ya, si la lactancia dura o sea si tú ya después de 4 horas teni las pechugas hinchadas porque no le has podido dar leche a tu hijo, te la tenía que sacar po y en qué trabajo te van a dejar sacarte leche... emm en muy pocos, en muy pocos, entonces eso es como preocupante $y$ teniendo la fortuna de trabajar media jornada no más po, porque si trabajara a jornada completa, más difícil todavia, en jornada completa tendrias casi que dejar de darle no más, porque como va a pasar 9 o 8 horas de no darle leche, a mi va a llegar un punto en que se me va a cortar la leche po entonces eso es como preocupante igual la vida laboral (P1).

El cumplimiento del rol de la "buena madre" se reconoce por las participantes como un proceso, muchas veces, tormentoso al cual deben adscribirse ya que el rol imperante de la madre así lo establece. Y, además, esto genera el surgimiento de sentimientos de culpa y rabia que tienen un impacto en la salud emocional de las mujeres madres.

[...] la lactancia materna es exquisita, es exquisito poder darle teta a tu hijo, porque te están mirando, están siempre mirándote, yo de repente le doy pecho a los dos juntos y es súper rico, porque los dos te están mirando, con una cara de amor y se te quita toda la rabia, la pena, se te quita todo, pero es como tortuoso el camino, porque aparte que igual te duelen los pezones, pero es como todo un proceso (P5).

[...] los retos tienen que ver con el descanso, tener que modificar la rutina, eem sobre todo el descanso porque en la noche que hay despertarse, emm que más. Emm... por ejemplo, si quiero salir, tengo que ajustarme que no pueden ser más de 4 horas, tengo que ser súper programada, tengo que dejarla tomada de leche para poder salir no más de 4 horas porque ya sé que me tiene que pedir de nuevo (P1).

[...] igual hay momentos en que cierta forma cuesta como aceptar que la vida cambia, no sé si un 100\% pero bastante y que los primeros años hay que dar mucho, hay que dar mucho por el hijo y uno de cierta forma se deja un poco de lado (P4). 
[...] el cuerpo te cambia, la demanda que te da como la... te quita libertad emm... (P2).

En ese sentido, los roles de género asociados a la maternidad y paternidad acompańan la precarización de las mujeres madres en su maternaje. A partir de ahí y de acuerdo con las entrevistadas, la labor del "padre" en este proceso se destaca como fundamental dentro de la participación ocupacional.

Yo siento que el bienestar que yo pueda tener y lo sigo confirmando que el bienestar de una mujer que está en proceso de lactancia no tiene que ver solamente con lo individual, indudablemente aqui tiene yo creo que el $50 \%$ si es que no es más el apoyo de tu pareja, yo creo que si a mi no me ha dado depresión es porque precisamente ha sido mi pareja quien se ha hecho cargo de los niños cuando llega del trabajo... si yo he podido cumplir y satisfacer mis necesidades de la vida diaria ha sido porque él me ha podido dar el apoyo y él ha podido asumir sus responsabilidades como padre en su totalidad, yo creo que eso es súper importante (P1).

Si, porque no solamente que te ayude con las cosas domésticas, si no que tengas con quien apoyarte en esos momentos de estrés o de angustia que tiene uno cuando está dando pecho, porque te angustia si no tienes much leche, estas muy sensible, entonces tienes que tener a alguien que te apoye, por ejemplo y que mejor si es tu pareja (P3).

Las narrativas descritas por las mujeres participantes en dicho estudio expresan la dificultad que se torna en muchas ocasiones el optar por la lactancia materna exclusiva como mecanismo único de alimentación. Asimismo, los discursos expresan una transición ocupacional compleja. Por un lado, las comprensiones sanitarias y socioculturales develan la importancia de la lactancia materna exclusiva, en tanto carácter nutritivo, inmunológico, emocional y psicológico. Por otro lado, y desde la óptica de las propias participantes y sus representaciones sociales atribuidas a este proceso como mujeres y madres, manifiestan la precariedad en la cual están llevando a cabo el proceso de lactancia y las implicaciones en lo cotidiano que esto tiene para sus vidas.

\section{Discusión}

Un debate sostenido se vislumbra en torno a los discursos sobre la lactancia materna exclusiva y la participación ocupacional que esta actividad implica en las mujeres madres que optan por este mecanismo de alimentación como único método. En ese sentido, se aprecian tres grandes categorías de análisis que lideran las reflexiones en torno a la lactancia materna: Lactancia materna exclusiva como ocupación, Lactancia materna exclusiva en el contexto socio-sanitario y reflexiones en torno a los desencuentros sobre los roles de mujer madre o madre mujer.

En relación con la Lactancia materna exclusiva como ocupación, es fundamental considerar de qué modo este aspecto responde a las transiciones, los ajustes y las adaptaciones del rol cultural que implica la actividad de lactar en las mujeres madres, y de qué modo esto afecta a la participación y el equilibrio ocupacional de las mismas (Blair, 2000). Su elección, y, por tanto, repercusión, se percibe en los cambios personales que constituye la llegada de los hijos e hijas en las perspectivas de vida de las mujeres siendo evidente el impacto que este 
fenómeno tiene en las diferentes áreas ocupacionales, como claramente se evidencia en la situación laboral, en el contexto y en las propias mujeres (Rodríguez et al., 2009). En ese sentido, los cambios que se destacan son los producidos en las actividades de la vida diaria, así como los tiempos que dedica la mujer madre a su alimentación, a su arreglo personal y a su higiene, adaptaciones que ocurren principalmente, en la primera etapa de la lactancia materna (Horne et al., 2005).

No obstante, estos cambios se relacionan con un aspecto temporal y transitorio en la vida de las mujeres, las cuales afirman que la participación diaria en actividades significativas como el ejercicio de la lactancia materna exclusiva, genera cambio en los patrones, sentimientos y en la participación ocupacional de sus vidas (Bar et al., 2016).

Esta transición ocupacional ocurrida en la primera etapa invita a las mujeres a probar y a aprender cosas nuevas a partir de sus propias capacidades, preferencias y valores (Kielhofner, 2011). En ese sentido, destacan emociones encontradas asociadas a ese proceso, tales como la ansiedad, la tristeza, la nostalgia y el cansancio, sentimientos derivados por la soledad, la escasez en la participación social y el cese de actividades de ocio y tiempo libre (Rodríguez et al., 2009; Gimeno, 2018).

Las emociones antes mencionadas, dan respuesta a un discurso hegemónico sobre la lactancia materna asociado al contexto actual en el cual se está maternando, en ese aspecto, la Lactancia materna exclusiva en el contexto socio-sanitario, se puede comprender desde una perspectiva cultural, como la transformación de roles y ocupaciones significativas vinculadas al rol de mujer y el cuidado personal que se alejan del concepto biomédico tradicional y se acercan a la influencia del ser sociocultural (Wilcock \& Towsend, 2009).

En ese aspecto, dentro de las características socio-sanitarias que contempla la lactancia materna asociado al discurso biomédico, se encuentra la sensación de miedo, emoción vinculada al temor que siente la mujer de estar alimentando satisfactoriamente o no a su hijo o hija. Esta emoción funciona incluso como predictor del mantenimiento de la lactancia materna en el tiempo (Shepherd et al., 2017; Komninou et al., 2017). Situación contraria se evidencia cuando los profesionales de la salud son comprensivos y guían el proceso de las mujeres, facilitando el acompañamiento en sus decisiones (Lumbiganon et al., 2011).

Asociado a lo anterior, se suma el castigo social existente por amamantar en público, el pudor que ello genera y la presión cultural existente, se impone aún desde el antiguo paradigma médico y la biologización de la mujer a amamantar, lo cual puede influenciar directamente en el éxito o fracaso de su permanencia (Komninou et al., 2017). Este paradigma rigidiza las prácticas médicas, ejemplo de ello es el establecimiento de horarios para lactar, perjudicando en muchas ocasiones, la adherencia de la lactancia materna exclusiva aumentando la percepción de estrés y descontento en las mujeres madres (Broche Cando et al., 2011). Asimismo, la adaptación ocupacional de las mujeres en el proceso de lactancia materna depende de aspectos fundamentales, tales como sus habilidades sociales y contar con una red de apoyo, tanto familiar como de la pareja, que entregue un soporte emocional durante todo el proceso (Horne et al., 2005; Pitonyak et al., 2015).

Situación socio-cultural que responde a las comprensiones de la última categoría Mujer madre o madre mujer. Así, la perspectiva de género devela la conflictiva situación que supone lactar hoy en día, en tanto implica la precarización del maternar y criar. 
A partir de ello, el acto de lactar genera sentimientos y pensamientos ambiguos, principalmente, relacionados con el significado emocional que la mujer madre atribuye a la lactancia y al cuidado del recién nacido en los términos que hoy en día son aceptados (Wilcock \& Towsend, 2009), es decir, el proveer de alimento y cuidado con constancia y dedicación (Pinto, 2007).

Esta situación invita a repensar en los roles de género de manera que problematice las condiciones estructurales (Lagarde, 1996) en las cuales hoy en día se desempeńa el ejercicio de lactar, así también, contribuye a interpelar los conocimientos desde espacios biomédicos no situados en las mujeres que vivencian este proceso y que se ven sometidas al escrutinio salubrista rígido que omite el carácter cultural que implica la lactancia materna (MassóGuijarro, 2013), perpetuando disparidades e injusticias ocupacionales en los procesos de salud/enfermedad de las mujeres madres (Pitonyak et al., 2015).

Por el contrario, cuando estas condiciones son consideradas y visibilizadas, avanzamos en comunidades comprometidas con los procesos de maternidad, lactancia y crianza, vitales para el desarrollo consciente y sostenible de una sociedad (Olza, 2013; Odent, 2014).

\section{Conclusiones}

Teniendo en cuenta la naturaleza y los resultados del presente estudio, el principal interés de esta investigación trata de comprender de qué modo la lactancia materna exclusiva impacta en la participación ocupacional de un grupo de mujeres chilenas. Por consiguiente, esta investigación no busca establecer relaciones causales directas entre estos dos fenómenos sino comprender, desde un punto de vista global, de qué modo la LME está relacionada con la participación ocupacional de modo que se pueda plantear dar respuestas ocupacionales antes de las demandas de las mujeres que están el proceso de la lactancia materna.

Desde el inicio de la lactancia materna se presentan facilitadores y dificultades sobre el proceso de participación en ocupaciones significativas. Esto afecta de forma directa a la identidad ocupacional de las mujeres madres, así como a su estado de salud y bienestar. No obstante, y de acuerdo con las participantes, es crucial comprender la perspectiva ocupacional de la lactancia materna. Esto permitirá plantear abordajes ocupacionales que promuevan la adaptación de la mujer madre en el proceso de lactancia y crianza, siendo consciente de las dinámicas hegemónicas que están presentes en dichos procesos. Asimismo, otro abordaje clave, de acuerdo con los resultados del presente estudio, podría ir enfocado a plantear abordajes ocupacionales de prevención y promoción de la salud y el bienestar para mujeres embarazadas que se plantean optar por la LME.

Junto a ello, es importante considerar de qué modo el acto de amamantar está relacionado con aspectos de participación e implicación en ocupaciones significativas para la mujer madre como son el cuidado personal y el ámbito laboral, entre otras áreas ocupacionales, afectadas por dicho fenómeno. Esta ruptura con la cotidianidad de las mujeres madres, así como la dificultad de participación ocupacional deben ser visibilizadas como un aspecto clave para el desarrollo comunitario social y personal de las mujeres. Empero, es importante no obviar que este proceso está inmerso en un entramado de significados socioculturales que no se pueden ni deben ignorar en el abordaje del complejo y atemporal fenómeno de la maternidad. 
Si bien la presente investigación se ha centrado principalmente en analizar el impacto de la lactancia materna en la participación ocupacional de un grupo de mujeres chilenas, sería importante para futuras líneas de investigación, profundizar de qué modo la lactancia materna afecta a la implicación y participación de ocupaciones significativas y cómo esto impacta en el vínculo entre la madre y el/la hijo/a. Otros aspectos interesantes a abordar son aquellos relacionados con explorar las posibilidades ocupacionales que la maternidad implica, reconociendo de qué modo la maternidad, como ocupación situada en un contexto sociocultural particular, puede ofrecer diferentes visiones y tipos de hacer y ser de acuerdo con un contexto sociohistórico. De este modo, comprender el impacto de la lactancia materna exclusiva en la participación ocupacional de las mujeres madres, permite identificar qué aspectos son claves para la prevención y promoción de la salud mental, sexual y reproductiva de las mujeres. A su vez, esta comprensión es clave para explorar las implicaciones en ocupaciones o actividades significativas teniendo en cuenta la perspectiva de género como base fundante para problematizar el rol de la mujer en los procesos de maternidad, lactancia y crianza. Escenario que nos invita a reflexionar sobre la emergencia de una salud ocupacional con enfoque crítico de género.

\section{Agradecimientos}

Mil gracias a todas las mujeres madres que participaron en esta investigación, a todas aquellas que se encuentran en periodo de lactancia materna y hacen visible una realidad que no todos quieren ver. A mis hijas Liera y Aliona por enseñarme el camino de la lactancia materna exclusiva con todo lo que ello implica y a Pavel por sostenerme en ese proceso. Gracias a Inma y Pía por su guía, entrega y compromiso en este artículo.

\section{Referencias}

Aguilar-Palafox, M. I., \& Fernández-Ortega, M. A. (2007). Lactancia materna exclusiva. Revista Facultad de Medicina UNAM, 50(4), 174-178.

American Association of Occupational Therapy - AOTA. (2014). Occupational therapy practice framework: domain and process. The American Journal of Occupational Therapy, 68(Suppl. 1), 1-48. http://dx.doi.org/10.5014/ajot.2014.682006.

Bar, M., Jarus, T., Wada, M., Rechtman, L., \& Noy, E. (2016). Male-to-female transitions: implications for occupational performance, health, and life satisfaction. Canadian Journal of Occupational Therapy, 83(2), 72-82.

Barrientos-Delgado, J. E. (2005). Calidad de vida, bienestar subjetivo: una mirada psicosocial. Santiago: Universidad Diego Portales.

Biblioteca del Congreso Nacional de Chile - BCN. (2016). Recuperado el 26 de diciembre de 2018, de https://www.bcn.cl/leyfacil/recurso/postnatal

Blair, S. (2000). The centrality of occupation during. British Journal of Occupational Therapy, 63(5), 231237. http://dx.doi.org/10.1177/030802260006300508.

Broche Cando, R. C., Sanchez Sarria, O. L., Rodriguez Rodriguez, D., \& Perez Ulloa, L. E. (2011). Factores socioculturales y psicológicos vinculados a la lactancia materna exclusiva. Revista Cubana de Medicina General Integral, 27(2), 254-260.

Cantero Garlito, P., Guajardo Cordoba, A., \& Sepulveda Prado, R. (2015). Terapia ocupacionaly exclusión social: hacia una praxis basada en los derechos humanos. Santiago: Segismundo. 
Centro de Investigación Periodística Chile - CIPER. (2016). Ciudad: Santiago. Recuperado el 8 de junio de 2019, de https:/ciperchile.cl/2016/07/14/cuida-el-ministerio-de-salud-de-chile-la-lactancia-materna/

Chale, L., Fenton, T., \& Kayange, N. (2016). Predictors of knowledge and practice of exclusive breastfeeding among health workers in Mwanza city, northwest Tanzania. BMC Nursing, 15(1), 1-8.

Coffey, A., \& Atkinson, P. (2003). Encontrar el sentido a los datos cualitativos: estrategias complementarias de investigación. Colombia: Editorial Universidad de Antioquía.

Dávila, A. (1995). Las perspectivas metodológicas cualitativa y cuantitativa en las ciencias sociales: Debate teórico e implicaciones praxeológicas. In J. M. Delgado \& J. Gutiérrez (Eds.), Métodos y técnicas cualitativas de investigación en ciencias sociales (pp. 69-83). Madrid: Síntesis.

Doble, S. E., \& Santha, J. C. (2008). Occupational well-being: rethinking occupational therapy outcomes. Revue Canadienne D'ergothérapie, 75(3), 184-190.

Fallon, V., Komninou, S., \& Bennet, K. (2016). The emotional and practical experiences of formula feeding mothers. Maternal and Child Nutrition, 13(2), 1-14.

Gimeno, B. (2018). La lactancia materna: política e identidad. España: Ediciones Cátedra.

Gutman, L. (2014). La maternidad y el encuentro con la propia sombra. Buenos Aires: Planeta.

Hidalgo, V., \& Menéndez, S. (2003). Ecoming parents: changes in the marital relationship during transition to parenthood. Infancia y Aprendizaje, 26(4), 469-483.

Horne, J., Corr, S., \& Earle, S. (2005). Becoming a mother: occupational change in first time motherhood. Journal of Occupational Science, 12(3), 176-183.

Kennedy, J., \& Davis, J. (2017). Clarifying the construct of occupational engagement for occupational therapy practice. OTJR: Occupation, Participation and Health, 37(2), 98-108.

Kielhofner, G. (2011). Modelo de ocupación humana: teoría y práctica. Buenos Aires: Panamericana.

Komninou, S., Fallon, V., Halford, J. C. G., \& Harrold, J. A. (2017). Differences in the emotional and practical experiences of exclusively breastfeeding and combination feeding mothers. Maternal and Child Nutrition, 13(3), 1-11.

Lagarde, M. (1996). El género en "género y feminismo": desarrollo humano y democracia. Madrid: Horas y Horas.

Llopis, M. (2015). Maternidades subversivas. Navarra: Txalaparta.

Loukas, K. (1992). Motherhood, occupational therapy, and feminism: weaving or unraveling the fibers of our lives? The American Journal of Occupational Therapy, 46(11), 1039-1041.

Lumbiganon, P., Martis, R., Laopaiboon, M., Festin, M. R., Ho, J. J., \& Hakimi, M. (2011). Antenatal breastfeeding education for increasing breastfeeding duration. Cochrane Database of Systematic Reviews, 11(11), CD006425.

Massó-Guijarro, E. (2013). Lactancia materna y revolución, o la teta como insumisión biocultural: calostro, cuerpo y cuidado. Dilemata, 5(11), 160-206.

Molina, M. E. (2006). Transformaciones histórico-culturales del concepto de maternidad y sus repercusiones en la identidad de la mujer. Psykhe, 15(2), 93-103.

Montero, I., Aparicio, D., Gómez-Beneyto, M., Moreno-Küstner, B., Reneses, B., Usall, J., \& VázquezBarquero, J. L. (2004). Género y salud mental en un mundo cambiante. Gaceta Sanitaria, 18(4, Suppl. 1), 175-181.

Moore, E., Bergman, N., Anderson, G. C., \& Medley, N. (2016). Early skin-to-skin contact for mothers and their healthy newborn infants. Cochrane Database of Systematic Reviews, 11, CD003519.

Odent, M. (2014). El parto y la evolución del homo sapiens. Londres: Pinter y Martin Ltd.

Olza, I. (2013). Lactivista. Madrid: Ob Stare.

Organización Mundial de la Salud - OMS. (2017). 10 datos sobre la lactancia materna. Recuperado el 25 de abril de 2017, de http://www.who.int/features/factfiles/breastfeeding/es

Organización Mundial de la Salud - OMS. (2019). Salud mental. Recuperado el 27 de agosto de 2017, de https://www.who.int/features/factfiles/mental_health/es/ 
Ovies-Carvallo, G., Santana, P. F., \& Padrón, D. R. S. (1999). Caracterización de la lactancia materna en un área urbana. Revista Cubana de Medicina General Integral, 15(1), 14-18.

Pérez, B. M. D., \& Moreno, H. A. (2017). Maternidades y lactancias desde una perspectiva de género. In E. Massó Guijarro (Ed.), Mamar: mythos y logos sobre lactancia humana (pp. 143-155). Madrid: Dilemata.

Pino, J. L., López, M. Á., Medel, A. P., \& Ortega, A. (2013). Factores que inciden en la duración de la lactancia materna exclusiva en una comunidad rural de Chile. Revista Chilena de Nutrición, 40(1), 48-54.

Pinto, F. (2007). Apego y lactancia natural. Revista Chilena de Pediatria, 78(1), 96-102.

Pitonyak, J. S., Mroz, T. M., \& Fogelberg, D. (2015). Expanding client-centred thinking to include social determinants: a practical scenario based on the occupation of breastfeeding. Scandinavian Journal of Occupational Therapy, 22(4), 277-282.

Poole, J., Willer, K., \& Mendelson, C. (2009). Occupation of motherhood: challenges for mothers with scleroderma. The American Journal of Occupational Therapy, 63(2), 214-219.

Rodríguez, M. D., Peña, J. V., \& Torío, S. (2009). La experiencia de la paternidad y la la maternidad: análisis del discurso de las creencias sobre la crianza y el cuidado infantil. Journal for the Study of Education and Developm, 32(1), 81-96.

Shepherd, L., Walbey, C., \& Lovell, B. (2017). The role of social-cognitive and emotional factors on exclusive breastfeeding duration. Journal of Human Lactation, 33(3), 606-613.

Spano-Nakano, A. M., Reis, M. C. G., Pereira, M. J. B., \& Gomes, F. A. (2007). Women's social space and the reference for breastfeeding practice. Revista Latino-Americana de Enfermagem, 15(2), 230-238.

Superintendencia de Seguridad Social - SUSESO. (2016). Protección a la maternidad en Chile: evolución del permiso postnatal parental a cinco años de su implementación 2011-2016. Santiago. Recuperado el 8 de junio de 2019, de https://www.suseso.cl/607/articles-19844_archivo_01.pdf

Taylor, S., \& Bodgan, R. C. (1987). Introducción a los métodos cualitativos de investigación. Barcelona: Paidós.

Vázquez Navarrete, M. L., Mogollón Pérez, A. S., Silva, M. R. F., Fernández de Sanmamed, M. J., Delgado Gallego, M. E., \& Vargas Lorenzo, I. (2006). Introducción a las técnicas cualitativas de investigación aplicadas en salud. Barcelona: Editorial Servei.

Wilcock, A., \& Towsend, E. (2009). Occupational justice. In B. A. B. Schell, G. Gillen \& M. E. Scafa (Eds.), Willard \& spackam ocuupational therapy (pp. 541-552). Philadelphia: Lippincot Williams \& Wikins.

\section{Contribución de los Autores}

Valeria Meline-Quińones ha participado en el diseńo de la investigación, en la recopilación de datos, análisis e interpretación de los datos, en la revisión crítica del manuscrito y finalmente, en la elaboración y aprobación del presente artículo. Pía Rodríguez-Garrido ha participado en el análisis, la interpretación de los datos, en la revisión crítica del manuscrito y finalmente, en la elaboración y aprobación del presente artículo.

Inmaculada Zango-Martin ha participado de manera conjunta con la primera autora en el diseńo de la investigación, el análisis e interpretación de los datos, en la revisión crítica del manuscrito y finalmente, en la elaboración y aprobación del presente artículo. Todos los autores aproban la version final del texto.

\section{Autor para la correspondencia}

Valeria Meline-Quiñones

e-mail: to.incluyendo@gmail.com 